\title{
Method for Converting Cone-Beam CT Values into Hounsfield Units for Radiation Treatment Planning
}

\author{
Tadanori Abe1,2, Kunihiko Tateoka, ${ }^{1,3}$, Yuichi Saito',4, Takuya Nakazawa1,5, Masaki Yano1, \\ Kensei Nakata ${ }^{6}$, Masanori Someya ${ }^{6}$, Masakazu Hori', Koichi Sakata6
}

\author{
${ }^{1}$ Depertment of Radiation Oncology and Medical Physics, Graduate School of Medicine, Sapporo Medical University, Sapporo, \\ Japan \\ ${ }^{2}$ Department of Radiology, KKR Sapporo Medical Center, Sapporo, Japan \\ ${ }^{3}$ Preparatory Office for Proton Therapy Center, Radiation Therapy Research Institute, Social Medical Corporation Teishinkai, \\ Sapporo, Japan \\ ${ }^{4}$ Department of Radiology, National Hospital Organization Hokkaido Cancer Center, Sapporo, Japan \\ ${ }^{5}$ Department of Radiology, Kushiro City General Hospital, Kushiro, Japan \\ ${ }^{6}$ Department of Radiation Oncology, School of Medicine, Sapporo Medical University, Sapporo, Japan \\ Email: abe.tadanori@sapmed.ac.jp
}

How to cite this paper: Abe, T., Tateoka, K., Saito, Y., Nakazawa, T., Yano, M., Nakata, K., Someya, M., Hori, M. and Sakata, K. (2017) Method for Converting Cone-Beam CT Values into Hounsfield Units for Radiation Treatment Planning. International Journal of Medical Physics, Clinical Engineering and Radiation Oncology, 6, 361-375. https://doi.org/10.4236/ijmpcero.2017.64032

Received: August 13, 2017

Accepted: October 1, 2017

Published: October 12, 2017

Copyright $\odot 2017$ by authors and Scientific Research Publishing Inc. This work is licensed under the Creative Commons Attribution International License (CC BY 4.0).

http://creativecommons.org/licenses/by/4.0/

\section{(c) (i) Open Access}

\begin{abstract}
Cone-beam CT (CBCT) images acquired during radiation treatment can be used to recalculate the dose distribution as well as to confirm the treatment location. However, it is difficult to obtain the electron densities (EDs) necessary for dose calculation from CBCT images because of the effects of scatter contamination during $\mathrm{CBCT}$ image acquisition. This paper presents a mathematical method for converting the pixel values of CBCT images (СВCT values) into Hounsfield units (HUs) of radiation treatment simulation CT (simCT) images for use in radiation treatment planning. СВCT values are converted into HUs by matching the histograms of the CBCT values with the histograms of the HUs for each slice via linear scaling of the CBCT values. For prostate cancer and head-and-neck cancer patients, the EDs obtained from converted CBCT values (mCBCT values) show good agreement with the EDs obtained from HUs, within approximately 3.0\%, and the dose calculated on the basis of CBCT images shows good agreement with the dose calculated on the basis of the simCT images, within approximately $2.0 \%$. Because the CBCT values are converted for each slice, this conversion method can account for variation in the CBCT values associated with differences in body size, body shape, and inner tissue structures, as well as in longitudinally displaced positions from the isocenter, unlike conventional methods that use electron density phantoms.
\end{abstract}


This method improves on conventional CBCT-ED conversion and shows considerable potential for improving the accuracy of radiation treatment planning using CBCT images.

\section{Keywords}

Cone-Beam Computed Tomography, Hounsfield Unit, Electron Density, Radiation Treatment Planning

\section{Introduction}

A cone-beam computed tomography (CBCT) unit is mounted on most linear accelerators. The effective use of CBCT images reduces patient setup inaccuracy by rigid image registration between daily $\mathrm{CBCT}$ images and radiation treatment simulation CT (simCT) images [1] [2]. However, in some cases, rigid image registration does not sufficiently account for anatomical changes in the target and organs at risk (OARs) that occur during some or all of the radiotherapy course [3]. Daily CBCT images can be used to recalculate the daily dose to a patient and facilitate the use of adaptive radiation therapy (ART), which accounts for daily anatomical changes in the target and the OARs [4].

In general, the dose calculations in a radiation treatment planning system (RTPS) are performed using an HU-ED or HU-PD conversion table that converts Hounsfield units (HUs) in simCT images into electron densities (EDs) or physical densities (PDs) [5] [6] [7]. However, it has been reported that CT values in CBCT images (CBCT values) may fluctuate, compared to HUs, because of scatter contamination, depending on the size, shape, and inner tissue structures [3] [8]-[13]. In addition, it has been reported that the CBCT values fluctuate with increasing distance from the center in the longitudinal direction [10]. Therefore, it is difficult to convert CBCT values directly into electron densities.

Several studies have reported that CBCT images can be used for dose calculations under such circumstances [8] [9] [10] [13] [14] [15] [16]. Yang et al. [9] mapped HUs in simCT images to the corresponding voxels in CBCT images after the simCT images were displaced and deformed into anatomical information in the CBCT images using deformable image registration (DIR). A bulk method has been proposed to assign general HUs (e.g., air $=-1000 \mathrm{HU}$, soft tissue $=0$ $\mathrm{HU}$, dense bone $=650 \mathrm{HU}$ ) or the mean HUs of the regions (air, soft tissue, bone, etc.) in the same patients to the same regions in the CBCT images [14] [15] [16]. Marchant et al. [17] created a shading map to correct CBCT values. These methods, however, involve a risk that the anatomical information in the CBCT images is lost.

To convert CBCT values directly into EDs or PDs, Richter et al. [10] generated CBCT-PD conversion tables using the PDs corresponding to the mean HUs of arbitrary regions of interest (ROIs) in simCT images and the mean CBCT values 
of the same ROIs in CBCT images of three different patient groups (head, chest, and pelvis). Similarly, $\mathrm{Hu}$ et al. [8] generated CBCT-PD conversion tables using the PDs corresponding to the mean HUs of 13 ROIs in simCT images and the mean CBCT values of the same ROIs in CBCT images acquired on the same days as the simCT images. Rong et al. [13] generated CBCT-ED conversion tables using electron density phantoms (EDPs) with sizes and shapes similar to those of the head, chest, and pelvis. These methods suggest that dose errors of more than $3 \%$ relative to dose calculations based on simCT images may occur when the patient groups or EDPs used to generate the conversion tables are different from the actual treatment size, shape, and inner tissue structures [10] [13]. In addition, the conversion tables do not account for variation in the scatter contamination with increasing distance from the isocenter (i.e., the center of the cone angle) in the longitudinal direction. Therefore, the conversion from CBCT values to electron densities and the calculated dose for regions including multiple treatment regions (e.g., head-and-neck regions including supraclavicular lymph nodes or truncal sites including the diaphragm) might be inaccurate.

In this paper, we examine a mathematical method of converting СBCT values into HUs. This approach aims to calculate the dose using an HU-ED conversion table, and we evaluate the application of this method to radiation treatment planning using a single photon beam.

\section{Materials and Methods}

\subsection{Conversion of CBCT Values into HUs}

Figure 1 shows an HU-ED conversion curve and a CBCT-ED conversion curve generated by using an electron density phantom (CIRS Electron Density Phantom Model 062 MA, Norfolk, VA, USA). The figure also shows histograms for which the vertical axis represents pixel counts having the same HUs or CBCT

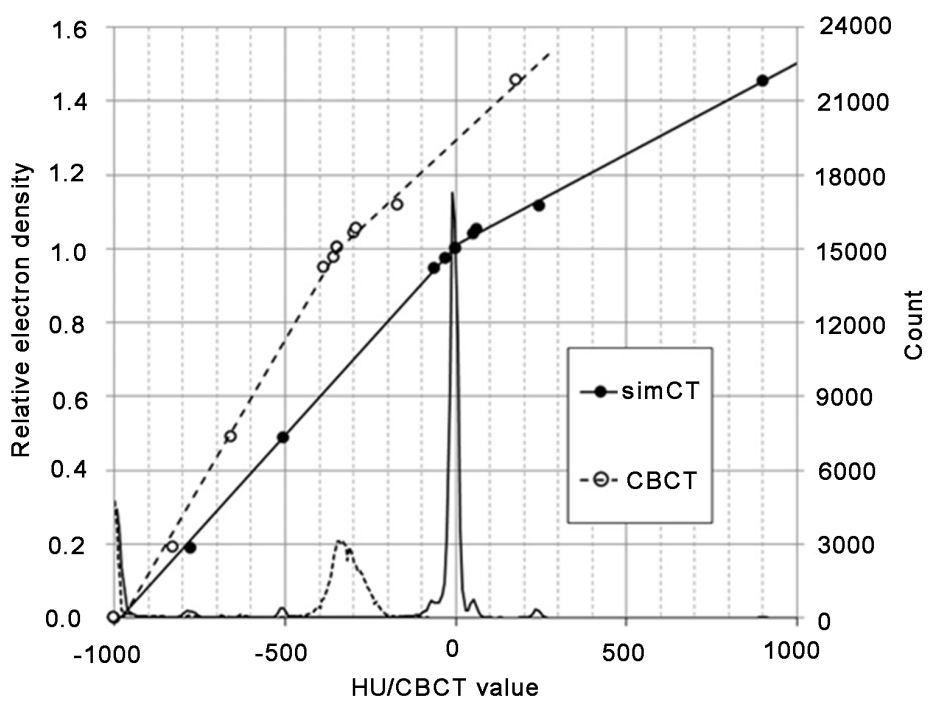

Figure 1. Electron density conversion curves and histograms acquired from simCT and CBCT images of electron density phantom. 
values. The simCT images were acquired using the GE Optima CT660 Pro (GE, Milwaukee, WI, USA), and the CBCT images were acquired using the Elekta Synergy XVI R4.0 (Elekta, Stockholm, Sweden). For the histograms of the simCT images, the positions of air and water were $-1000 \mathrm{HU}$ and $0 \mathrm{HU}$, respectively, based on HU calibration. The positions and heights of the histograms of the CBCT images were different from those of the simCT images because the CBCT values were affected by scatter contamination. The HU-ED and CBCT-ED conversion curve forms two approximate straight lines near the folding point of the electron density of water [10] [11]. We converted the CBCT values into HUs by linear scaling of the CBCT values for the soft tissue and bone regions. СBCT values were converted into HUs for five prostate cancer patients and three head-and-neck cancer patients. These patients were selected at random regardless of size, age, and the tumor clinical stages. SimCT images were acquired using the helical mode at settings of $120 \mathrm{kV}, 400 \mathrm{~mA}$, and a $50-\mathrm{cm}$ field of view (FOV). CBCT images were acquired at settings of $120 \mathrm{kV}$ and $40 \mathrm{~mA}$, using an $\mathrm{M} 20$ collimator cassette with a $41-\mathrm{cm}$ FOV and $27.67-\mathrm{cm}$ longitudinal FOV. A bowtie filter was used to obtain clear body contours.

The first step for the conversion of $\mathrm{CBCT}$ values into HUs is to establish a correspondence between the spatial locations of the pixels in the simCT and $\mathrm{CBCT}$ images (resampling). The three-dimensional bone information in the simCT and $\mathrm{CBCT}$ images were matched by implementing bone-based rigid image registration between the simCT and $\mathrm{CBCT}$ images. The slice thickness and matrix size of the СBCT images were matched with those of the simCT images. We refer to the CBCT images created in this step as resampled CBCT (rCBCT) images. The bone-based rigid image registration and $\mathrm{rCBCT}$ image generation were performed using Velocity AI (Velocity Medical Solutions, Atlanta, GA, USA).

The rCBCT values were converted into HUs according to the following steps.

1) The simCT images and rCBCT images of the same patient were imported into an in-house software program.

2) The soft tissue and bone regions for the simCT and CBCT images were divided because the HU-ED and CBCT-ED conversion tables have different slopes above the electron density region of water, as shown in Figure 1. If there was some noise that has high CBCT values such as streak artifacts around the bone, this noise was eliminated from the bone region using erosion and dilation operators. (This step does not involve the elimination of streak artifacts.)

3) For the soft tissue and bone regions in each slice of the rCBCT images, linear scaling of the rCBCT values was performed to match the histograms of the rCBCT values and the simCT values to the greatest extent possible. The CBCT images created using this conversion method are referred to as modified CBCT (mCBCT) images.

The linear scaling was performed by assigning arbitrary values to $a$ and $b$ in Equation (1).

$$
m C B C T \text { value }=a \times r C B C T \text { value }+b
$$


where $a$ and $b$ are arbitrary values at 0.1 intervals and were chosen to match both histograms.

To evaluate the consistency of the histograms between the HUs and mCBCT values, the sum of the squared differences (SSD) of the pixel counts of the histogram were calculated using Equation (2) and Equation (3).

$$
\begin{aligned}
& \text { Soft tissue region: } \sum_{i=-1000}^{\text {threshold }}\left(\text { Count }_{\operatorname{simCT}(i)}-\text { Count }_{m C B C T(i)}\right)^{2} \\
& \text { Bone region: } \sum_{i=\text { threshold }}^{1500}\left(\text { Count }_{\text {simCT }(i)}-\text { Count }_{m C B C T(i)}\right)^{2}
\end{aligned}
$$

where $i$ denotes the HU or mCBCT value, threshold is the threshold value for dividing the soft tissue and bone regions in $\mathrm{CBCT}$ images (the threshold is equal to the value of the peak-end corresponding to the soft tissue in the histogram in most cases), Count $\operatorname{simCT(i)}_{i}$ is the pixel count with $\mathrm{HU}=i$, and Count $_{m C B C T(i)}$ is the pixel count with an $\mathrm{mCBCT}$ value $=i$ calculated using Equation (1).

The $a$ and $b$ values were given to Equation (1), and the SSD for the soft tissue and the bone regions was calculated using Equation (2) and Equation (3). These calculations were iteratively performed until the minimum SSD was acquired.

4) The mCBCT images created were saved as DICOM files.

At present, the application of a sequence of these steps (from scanning the $\mathrm{CBCT}$ images to creating the mCBCT images) takes approximately $30 \mathrm{~min}$ per 100 images to complete on a general-purpose computer with a $3.40 \mathrm{GHz}$ Intel Core i7 CPU and 12.0 GB RAM.

\subsection{Evaluation of mCBCT Values}

In the evaluation of the $\mathrm{mCBCT}$ values, the relative electron densities converted from the mCBCT values $\left(\mathrm{RED}_{\mathrm{mCBCT}}\right)$ were compared with the relative electron densities converted from the HUs $\left(\mathrm{RED}_{\text {simCT }}\right)$ for the pelvic and head-and-neck regions. The $\mathrm{RED}_{\mathrm{mCBCT}}$ and $\mathrm{RED}_{\text {simCT }}$ values were converted using the HU-ED conversion table generated from the electron density phantom (Figure 2(a)).

To compare this method with the conventional method, the relative electron densities converted from the rCBCT values $\left(\mathrm{RED}_{\mathrm{rCBCT}}\right)$ were evaluated. The rCBCT values were converted into $\mathrm{RED}_{\mathrm{rCBCT}}$ values using the method described by Rong [13], which involves the use of CBCT-ED conversion tables (Figure 2(c)) generated from CBCT images of electron density phantoms of the pelvis (Figure 2(a)) and head (Figure 2(b)).

For the pelvic region, the ROIs evaluated were the prostate, adipose, muscle, and bone (Figure 3). For the head-and-neck regions, the ROIs evaluated were the parotid gland, muscle, spinal cord, and bone (Figure 4). The ROIs of each region were set in the axial images at the isocenter and longitudinally displaced positions (off-center).

\subsection{Evaluation of Calculated Dose}

In the evaluation of the dose calculations using the $\mathrm{mCBCT}$ images, treatment plans based on the simCT and СBCT images were developed using a single 


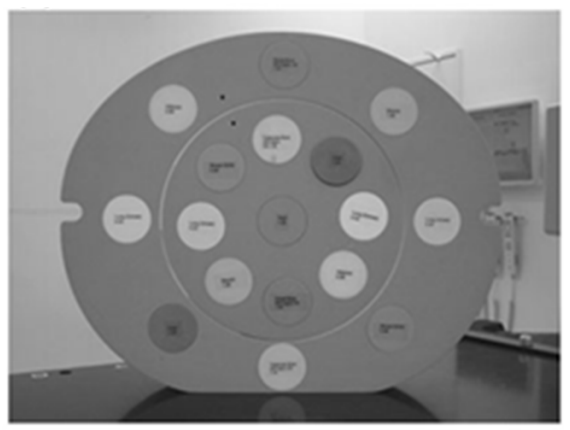

(a)

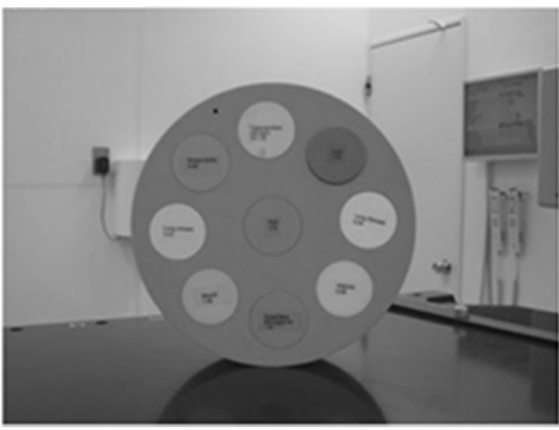

(b)

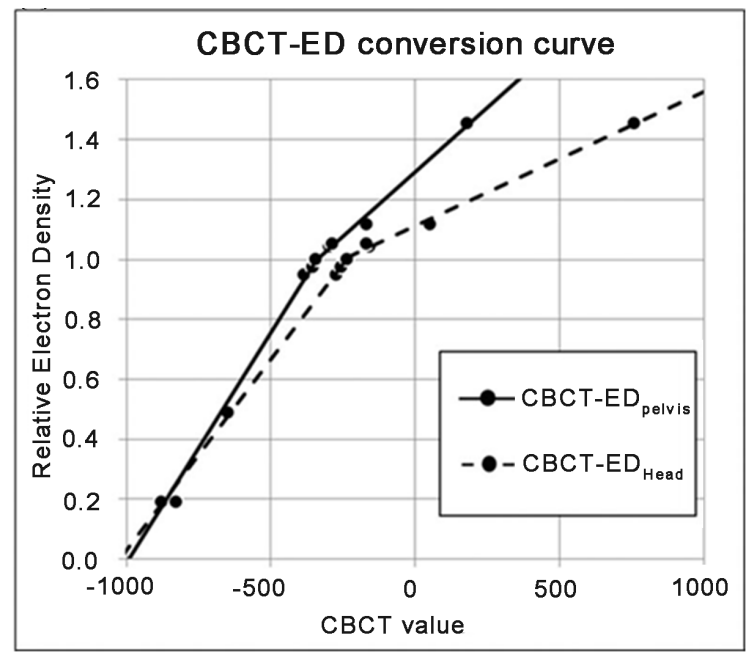

(c)

Figure 2. Electron density phantoms of the (a) pelvis and (b) head region, and (c) CBCT-ED conversion curves created from the electron density phantoms of each region: CBCT-ED $\mathrm{Head}_{\text {and }}$ CBCT-ED $\mathrm{Pelvis}_{\text {. }}$
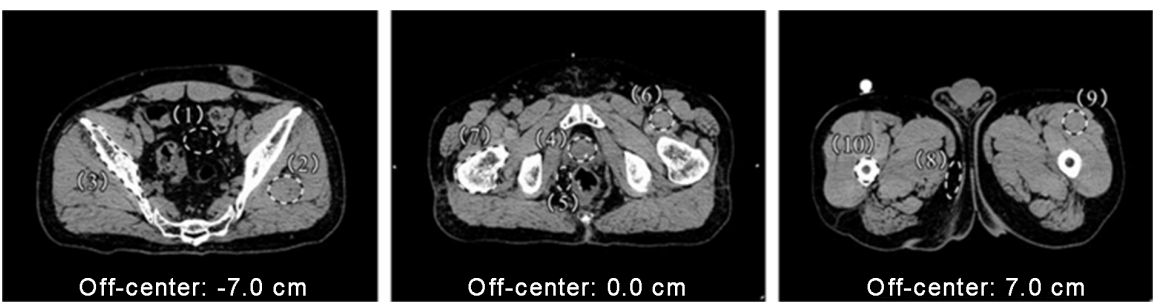

Figure 3. Axial simCT images of a prostate cancer patient and ROIs: 1) visceral fat, 2) gluteus maximus muscle, 3) iliac bone, 4) prostate, 5) visceral fat, 6) iliopsoas muscle, 7) thigh bone, 8) subcutaneous adipose, 9) biceps femoris, and 10) thigh bone.

anterior photon beam passing through the slices shown in Figure 3 and Figure 4. The photon energy was $10 \mathrm{MV}$ for the prostate cases and $4 \mathrm{MV}$ for the head-and-neck cases. All of the plans had beams at the same isocenter, with 100 monitor units (MUs). The calculated dose profiles based on the CBCT images for the slices shown in Figure 3 and Figure 4 were compared with the dose profiles based on the simCT images. The differences in the dose distributions between the CBCT plans and the simCT plans were analyzed using gamma 

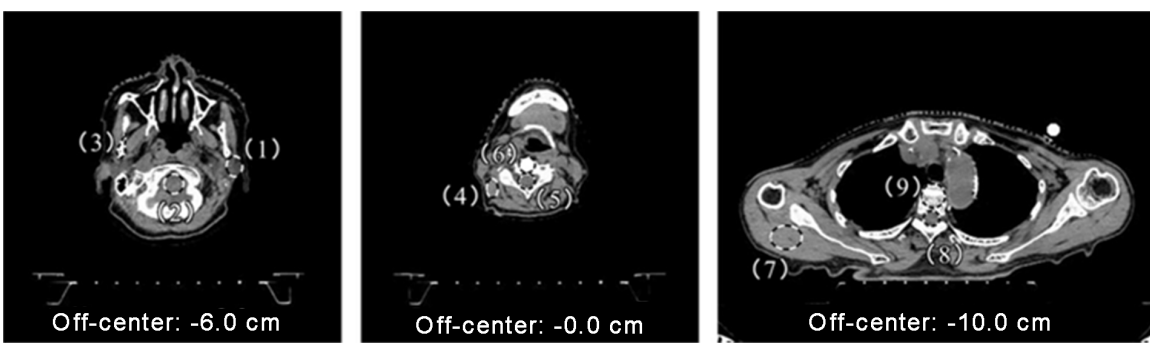

Figure 4. Axial simCT images of a head-and-neck cancer patient and ROIs: 1) parotid gland, 2) spinal cord, 3) mandibular bone, 4) trapezius muscle, 5) spinal cord, 6) vertebra, 7) infraspinatus muscle, 8) spinal cord, and 9) vertebra.

analysis. Gamma analysis was performed with a $2 \%$ dose-difference (DD) and 2-mm distance-to-agreement (DTA) criteria using commercial software (Verisoft, Version 4.0, PTW, Freiburg, Germany).The RTPS used in this study was Eclipse ver. 11 (Varian Medical Systems, Palo Alto, CA, USA), and the dose calculation algorithm used was the analytical anisotropic algorithm (AAA). For the dose calculation, the HU-ED conversion table described in the previous section was used for the treatment plans based on the simCT and mCBCT images, and CBCT-ED conversion tables were used for the treatment plans based on the rCBCT images.

\section{Results}

\subsection{Conversion of CBCT Values into HUs}

The simCT, $\mathrm{rCBCT}$, and $\mathrm{mCBCT}$ images of a prostate cancer patient and a head-and-neck cancer patient are shown in Figure 5 and Figure 6, respectively. When these images were displayed with the same window level (WL) and window width (WW), the simCT and $\mathrm{mCBCT}$ images had a subequal dynamic range. However, shading artifacts that reduce the $\mathrm{MCBCT}$ values were observed in the posterior regions of all of the slices for the prostate cancer patient as well as the 10.6- $\mathrm{cm}$ off-center slice for the head-and-neck cancer patient. In addition, streak artifacts that increase the $\mathrm{mCBCT}$ values were observed around the bone in all of the $\mathrm{mCBCT}$ images.

The histograms of the HUs and CBCT values of the axial images of the isocenter $(0.0 \mathrm{~cm}$ off-center) slice of the prostate cancer patient (Figures $5(\mathrm{~d})$-(f)) are shown in Figure 7. The two peaks (approximately $-100 \mathrm{HU}$ and $50 \mathrm{HU}$ ) in the soft tissue region of the histogram of the HUs (the solid line) correspond to adipose and muscle tissue, respectively. The peak in the soft tissue region of the histogram of the CBCT values (the dashed line) was approximately -350 , which made it difficult to discern adipose and muscle tissue. The peak in the soft tissue region of the histogram of the $\mathrm{mCBCT}$ values (the dotted line) was nearly equal to that of the histogram of the HUs. However, it was difficult to discern adipose and muscle tissue. Similar results were obtained for the histograms of the other slices for the other prostate cancer patients and the head-and-neck cancer patient. 


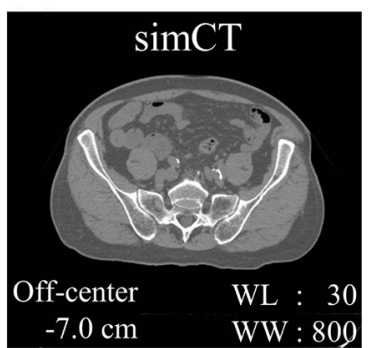

(a)

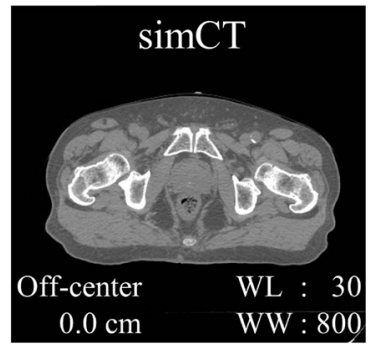

(d)

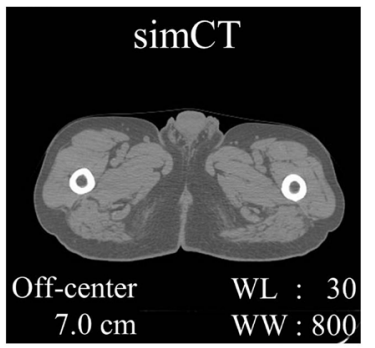

(g)

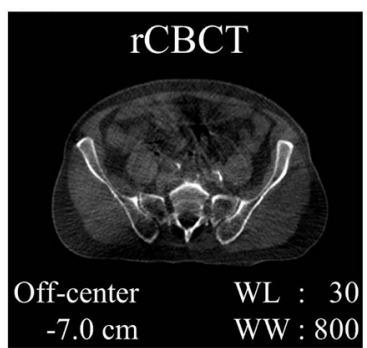

(b)

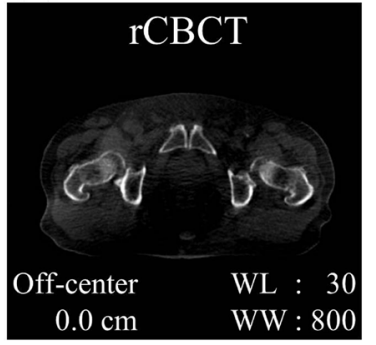

(e)

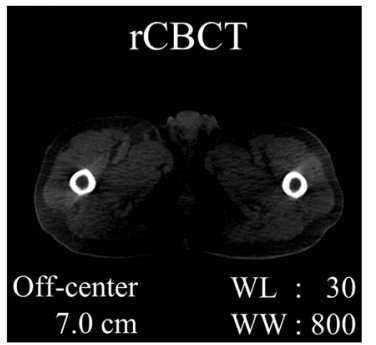

(h)

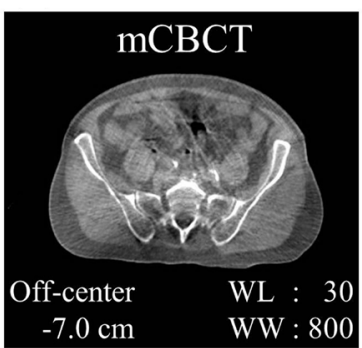

(c)

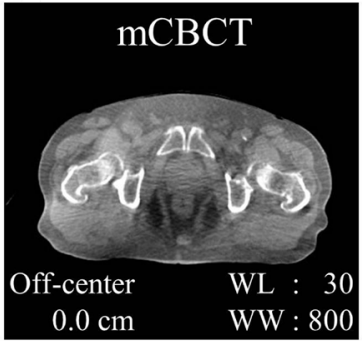

(f)

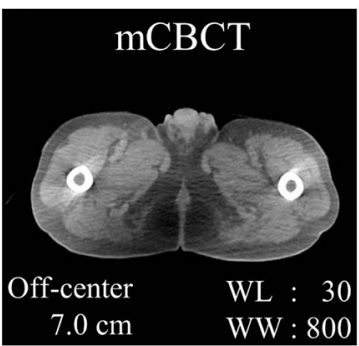

(i)

Figure 5. Axial images of a prostate cancer patient. All images are shown with the same window level (WL) and window width (WW).

\subsection{Evaluation of mCBCT Values}

Table 1 shows the results for the ten ROIs (see Figure 3) that were evaluated in the isocenter $(0.0 \mathrm{~cm}$ off-center $)$ and off-center slices for the five prostate cancer patients. The differences in $\mathrm{RED}_{\mathrm{rCBCT}}$ for the off-center slices were larger than those for the isocenter slices. The standard deviations (SD) of the differences in $\mathrm{RED}_{\mathrm{rCBCT}}$ for the slices were large because one CBCT-ED conversion table cannot take into account differences in patients' body sizes. In contrast, the differences in $\mathrm{RED}_{\mathrm{mCBCT}}$ for the off-center slices were nearly equal to the differences in those for the isocenter slice (the difference in the total mean \pm one SD was -0.34 $\pm 0.97 \%)$.

Table 2 shows the results for the nine ROIs (see Figure 4) that were evaluated in the isocenter and off-center slices for the three head-and-neck cancer patients. As in the case of the prostate cancer patients, the differences in $\mathrm{RED}_{\mathrm{mCBCT}}$ for the off-center slices were larger than those for the isocenter slices. The differences in $\mathrm{RED}_{\mathrm{mCBCT}}$ for the off-center slices were nearly equal to the differences in those for the isocenter slice (the difference in the total mean \pm one SD was $-0.15 \pm$ $1.43 \%$ ), except for the muscle for the $10.0-\mathrm{cm}$ off-center slice. 


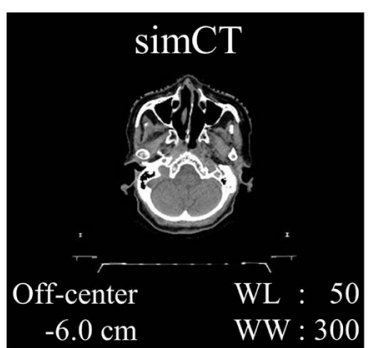

(a)

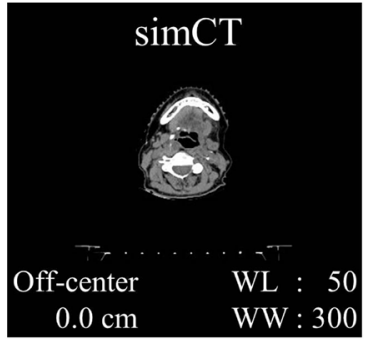

(d)

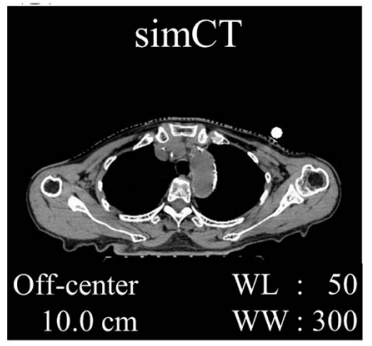

(g)

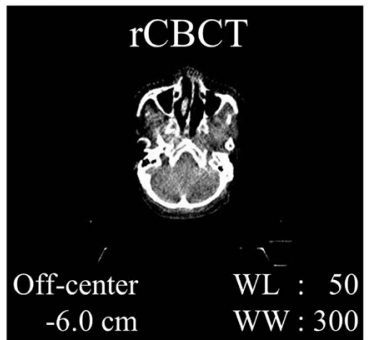

(b)

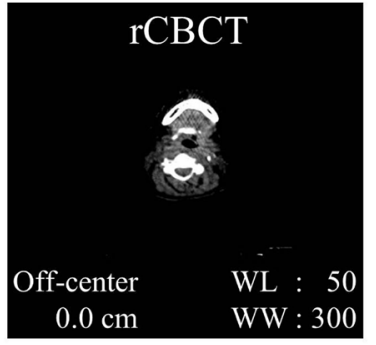

(e)

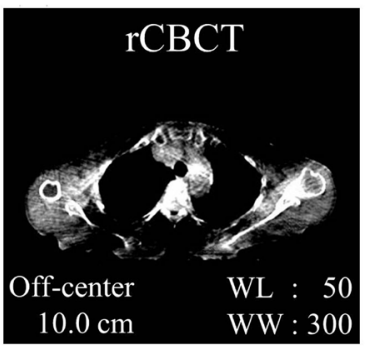

(h)

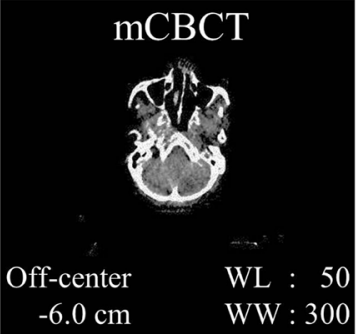

(c)

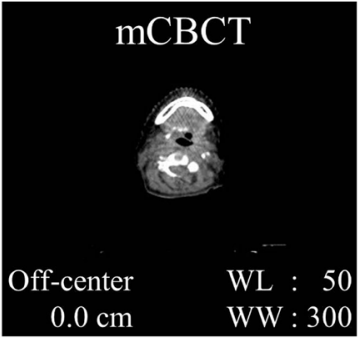

(f)

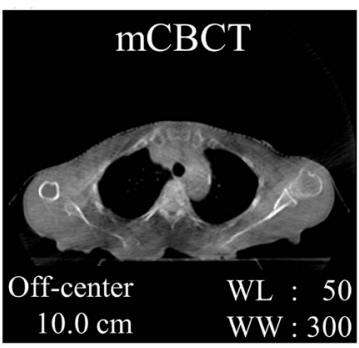

(i)

Figure 6. Axial images of a head-and-neck cancer patient. All images are shown with the same window level (WL) and window width (WW).

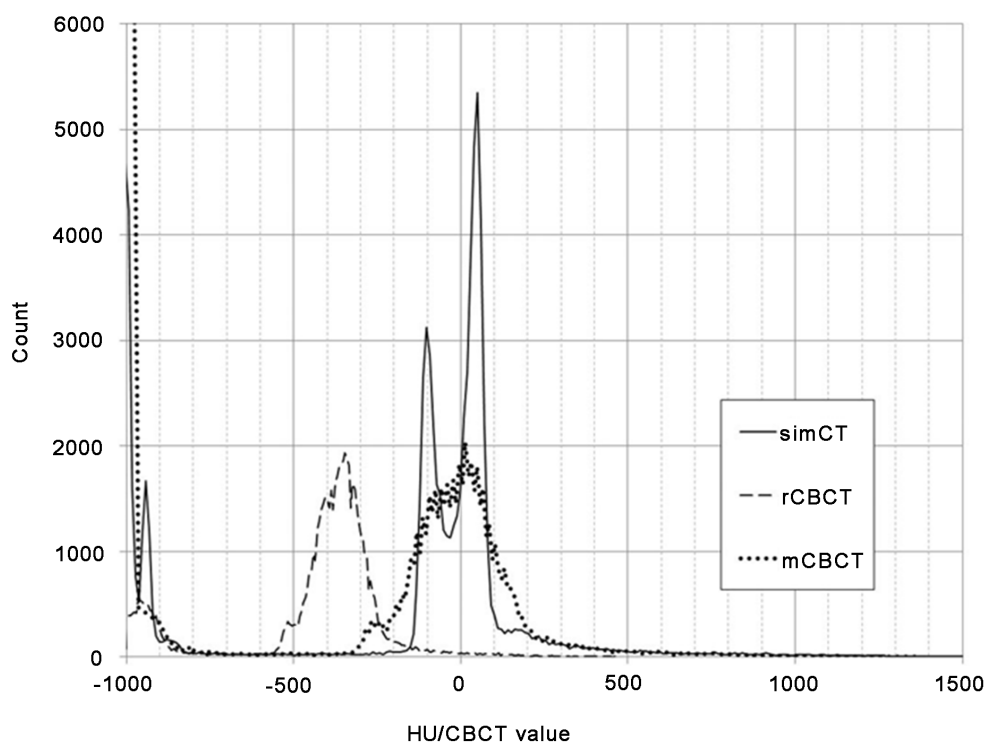

Figure 7. Histograms of $\mathrm{HU}$ and $\mathrm{CBCT}$ values acquired from simCT, rCBCT, and $\mathrm{mCBCT}$ images for the prostate cancer patient. The two peaks in the soft tissue region of the simCT images (solid line) correspond to adipose and muscle tissue. 
Table 1. Comparison of CBCT values and differences in RED for each ROI of the prostate cancer patients.

\begin{tabular}{|c|c|c|c|c|c|}
\hline \multirow[b]{2}{*}{ ROI No. } & \multirow{2}{*}{$\begin{array}{c}\operatorname{simCT} \\
\mathrm{HU}\end{array}$} & \multicolumn{2}{|c|}{$\mathrm{rCBCT}$} & \multicolumn{2}{|c|}{$\mathrm{mCBCT}$} \\
\hline & & rCBCT value & $\begin{array}{l}\text { \% difference in } \\
\text { RED }_{\mathrm{rCBCT}}\end{array}$ & mCBCT value & $\begin{array}{l}\% \text { difference in } \\
\text { RED }_{\text {mСвст }}\end{array}$ \\
\hline \multicolumn{6}{|c|}{ off-center $-7.0 \mathrm{~cm}$ (superior) } \\
\hline 1 & $-87 \pm 29$ & $-493 \pm 24$ & $-14.74 \% \pm 3.43 \%$ & $-95 \pm 36$ & $-0.83 \% \pm 1.50 \%$ \\
\hline 2 & $54 \pm 7$ & $-312 \pm 33$ & $-2.43 \% \pm 2.78 \%$ & $46 \pm 13$ & $-0.38 \% \pm 0.48 \%$ \\
\hline 3 & $415 \pm 55$ & $-40 \pm 152$ & $3.21 \% \pm 11.22 \%$ & $393 \pm 57$ & $-0.92 \% \pm 1.05 \%$ \\
\hline$($ mean $\pm 1 S D)$ & & & $(-4.66 \% \pm 10.09 \%)$ & & $(-0.71 \% \pm 1.04 \%)$ \\
\hline \multicolumn{6}{|c|}{ off-center $0.0 \mathrm{~cm}$ (center) } \\
\hline 4 & $36 \pm 8$ & $-329 \pm 46$ & $-2.26 \% \pm 3.88 \%$ & $29 \pm 7$ & $-0.36 \% \pm 0.23 \%$ \\
\hline 5 & $-97 \pm 23$ & $-413 \pm 44$ & $-0.86 \% \pm 5.90 \%$ & $-99 \pm 20$ & $-0.26 \% \pm 0.65 \%$ \\
\hline 6 & $59 \pm 6$ & $-267 \pm 55$ & $1.24 \% \pm 4.57 \%$ & $67 \pm 14$ & $0.37 \% \pm 0.40 \%$ \\
\hline 7 & $286 \pm 72$ & $-147 \pm 83$ & $0.76 \% \pm 7.53 \%$ & $301 \pm 69$ & $0.64 \% \pm 1.01 \%$ \\
\hline$($ mean $\pm 1 S D)$ & & & $(-0.28 \% \pm 5.37 \%)$ & & $(0.10 \% \pm 0.73 \%)$ \\
\hline \multicolumn{6}{|c|}{ off-center $7.0 \mathrm{~cm}$ (inferior) } \\
\hline 8 & $-96 \pm 15$ & $-458 \pm 77$ & $-8.76 \% \pm 11.06 \%$ & $-111 \pm 18$ & $-1.62 \% \pm 0.64 \%$ \\
\hline 9 & $61 \pm 5$ & $-287 \pm 67$ & $-0.05 \% \pm 4.98 \%$ & $69 \pm 3$ & $0.37 \% \pm 0.32 \%$ \\
\hline 10 & $772 \pm 223$ & $146 \pm 123$ & $2.51 \% \pm 11.20 \%$ & $763 \pm 240$ & $-0.37 \% \pm 0.78 \%$ \\
\hline$($ mean $\pm 1 S D)$ & & & $(-2.10 \% \pm 10.14 \%)$ & & $(-0.54 \% \pm 1.02 \%)$ \\
\hline \multicolumn{3}{|c|}{ Total mean $\pm 1 \mathrm{SD}$} & $-2.14 \% \pm 8.54 \%$ & & $-0.34 \% \pm 0.97 \%$ \\
\hline
\end{tabular}

Table 2. Comparison of CBCT values and differences in RED for each ROI of the head-and-neck cancer patients.

\begin{tabular}{|c|c|c|c|c|c|}
\hline \multirow[b]{2}{*}{ ROI No. } & \multirow{2}{*}{$\begin{array}{c}\operatorname{simCT} \\
\mathrm{HU}\end{array}$} & \multicolumn{2}{|c|}{$\mathrm{rCBCT}$} & \multicolumn{2}{|c|}{$\mathrm{mCBCT}$} \\
\hline & & $\begin{array}{l}\text { rCBCT } \\
\text { value }\end{array}$ & $\begin{array}{l}\text { \% difference in } \\
\operatorname{RED}_{\mathrm{rCBCT}}\end{array}$ & mCBCT value & $\begin{array}{l}\text { \% difference in } \\
\operatorname{RED}_{\mathrm{mCBCT}}\end{array}$ \\
\hline \multicolumn{6}{|c|}{ off-center $-6.0 \mathrm{~cm}$ (superior) } \\
\hline 1 & $15 \pm 5$ & $-116 \pm 38$ & $4.70 \% \pm 1.77 \%$ & $-1 \pm 1$ & $-1.13 \% \pm 0.58 \%$ \\
\hline 2 & $41 \pm 6$ & $-77 \pm 44$ & $4.88 \% \pm 1.67 \%$ & $40 \pm 21$ & $-0.05 \% \pm 0.97 \%$ \\
\hline 3 & $513 \pm 37$ & $507 \pm 44$ & $3.79 \% \pm 1.37 \%$ & $537 \pm 43$ & $0.93 \% \pm 0.29 \%$ \\
\hline$($ mean $\pm 1 S D)$ & & & $(4.46 \% \pm 1.49 \%)$ & & $(-0.08 \% \pm 1.07 \%)$ \\
\hline \multicolumn{6}{|c|}{ off-center $0.0 \mathrm{~cm}$ (center) } \\
\hline 4 & $57 \pm 11$ & $-130 \pm 28$ & $2.03 \% \pm 0.86 \%$ & $51 \pm 13$ & $-0.28 \% \pm 0.67 \%$ \\
\hline 5 & $40 \pm 5$ & $-114 \pm 27$ & $3.52 \% \pm 1.02 \%$ & $25 \pm 11$ & $-0.72 \% \pm 0.76 \%$ \\
\hline 6 & $441 \pm 54$ & $393 \pm 54$ & $3.12 \% \pm 1.12 \%$ & $465 \pm 52$ & $0.96 \% \pm 0.25 \%$ \\
\hline$($ mean $\pm 1 \mathrm{SD})$ & & & $(2.89 \% \pm 1.10 \%)$ & & $(-0.01 \% \pm 0.92 \%)$ \\
\hline \multicolumn{6}{|c|}{ off-center $10.0 \mathrm{~cm}$ (inferior) } \\
\hline 7 & $41 \pm 5$ & $-258 \pm 11$ & $-5.96 \% \pm 1.44 \%$ & $-15 \pm 18$ & $-3.19 \% \pm 0.32 \%$ \\
\hline 8 & $38 \pm 6$ & $-260 \pm 12$ & $-6.03 \% \pm 1.54 \%$ & $57 \pm 9$ & $0.95 \% \pm 0.19 \%$ \\
\hline 9 & $176 \pm 18$ & $-133 \pm 46$ & $-3.59 \% \pm 1.19 \%$ & $201 \pm 11$ & $1.16 \% \pm 0.39 \%$ \\
\hline$($ mean $\pm 1 S D)$ & & & $(-5.19 \% \pm 1.71 \%)$ & & $(-0.36 \% \pm 2.14 \%)$ \\
\hline \multicolumn{3}{|c|}{ Total mean $\pm 1 \mathrm{SD}$} & $0.72 \% \pm 4.53 \%$ & & $-0.15 \% \pm 1.43 \%$ \\
\hline
\end{tabular}




\subsection{Evaluation of Calculated Dose}

For the evaluation of dose calculations using the $\mathrm{CBCT}$ images, the treatment plans generated on the basis of the $\mathrm{mCBCT}$ and $\mathrm{rCBCT}$ images (the mCBCT plans and $\mathrm{rCBCT}$ plans, respectively) were compared with the treatment plans generated on the basis of the simCT images (the simCT plans). Figure 8 shows the lateral dose profiles and dose differences for the isocenter and off-center slices obtained using the anterior single beam. In the field for this prostate case, the rCBCT plan agreed with the simCT plan between $2.0 \%$ and $0.0 \%$ for the isocenter slice. For the $-7.0-\mathrm{cm}$ off-center slice, however, the rCBCT plan showed dose differences of up to $-4.6 \%$ (the maximum dose difference for the other prostate case was approximately 6.5\%). The dose difference in the rCBCT plan for the head-and-neck case was approximately $-4 \%$, despite the isocenter slice. The dose differences for the -6.0 - $\mathrm{cm}$ off-center slice showed an under dose (minimum $-6.9 \%$ ), and those for the 10.0 -cm off-center slice showed overdosing (between $2 \%$ and $6 \%$ ). On the other hand, the $\mathrm{mCBCT}$ plan resulted in dose differences that agreed to within $2 \%$ with the simCT plan for all of the slices. For
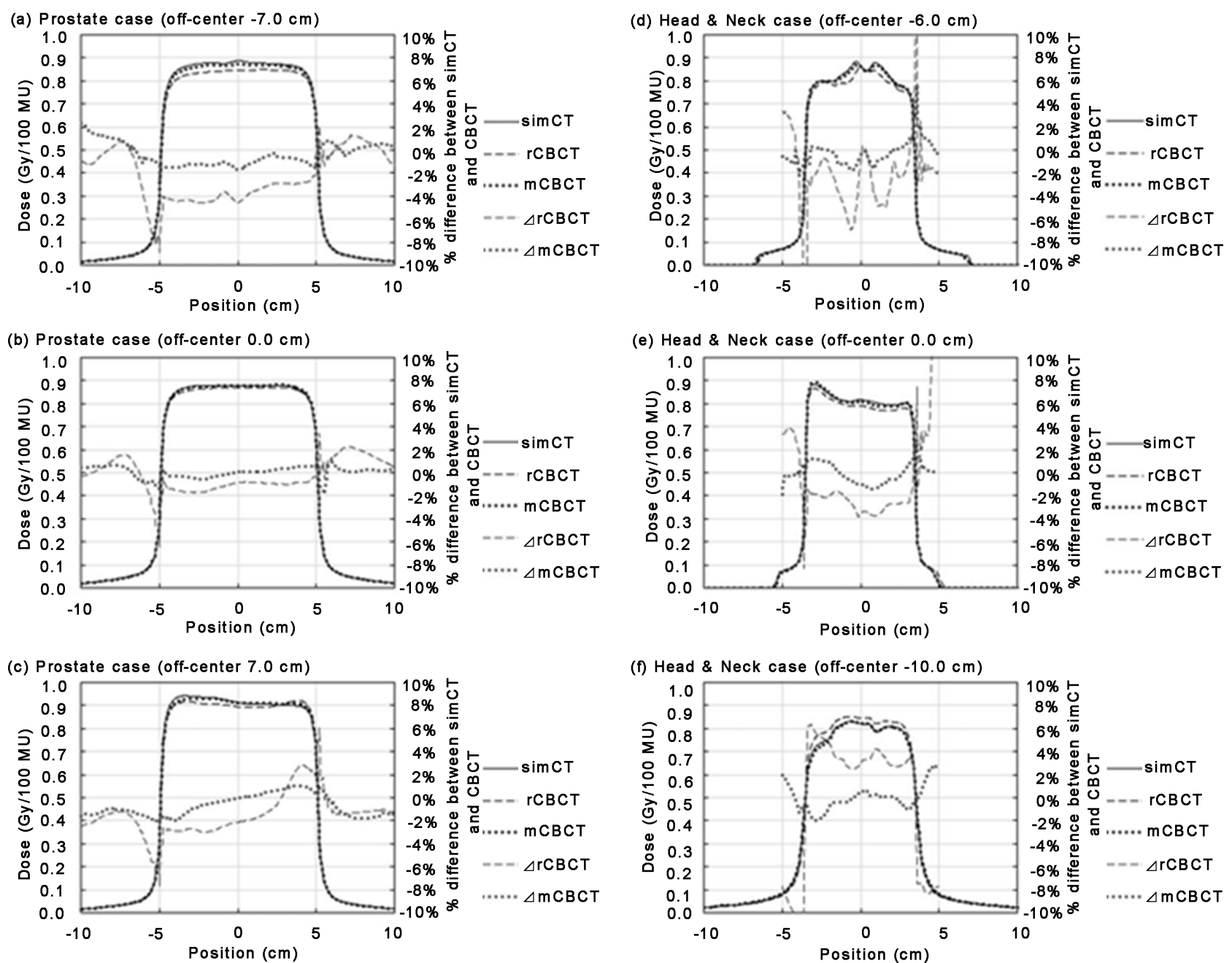

Figure 8. Comparison of the dose profiles for (a)-(c) prostate cancer patient case and (d)-(f) head-and-neck cancer patient case. 
the other prostate cases, the results in the dose differences of $\mathrm{rCBCT}$ and $\mathrm{mCBCT}$ plans were similar to those for this case.

Table 3 shows the results of gamma analysis for prostate and head-and-neck cancer patients. The gamma passing rates were calculated with a criterion of $2 \% / 2-\mathrm{mm}$ and a threshold of $20 \%$. For most of the rCBCT plans, the passing rates were less than $90 \%$ except for the axial-center slices. The passing rates for most of the mCBCT plans were better than approximately $95 \%$, and there was no significant difference between the isocenter slices and off-center slices.

\section{Discussion}

The CBCT mounted on linear accelerator has become a valuable tool for adaptive radiation therapy. In Figure 1 and Figure 7, however, the position of the mode for the CBCT values and HUs was different because the CBCT system used in this study does not provide an HU calibration tool. Previous studies involving direct conversion of CBCT values into electron densities used CBCT-ED conversion tables generated from $\mathrm{CBCT}$ images of electron density phantoms [10] [13]. However, for treatment sites with different sizes and inner tissue structures than the electron density phantom, differences between $\mathrm{RED}_{\mathrm{rCBCT}}$ and $\mathrm{RED}_{\text {simCT }}$ were observed in this study. As shown in Table 1, for the prostate cancer cases, the standard deviation of the differences between $\mathrm{RED}_{\mathrm{rCBCT}}$ and $\mathrm{RED}_{\text {simCT }}$ was large, at $8.54 \%$, while the mean difference was only $-2.14 \%$. This illustrates the differences in body size and inner tissue structures between the patients and the electron density phantom. The standard deviations of the differences in $\mathrm{RED}_{\mathrm{rCBCT}}$ for the off-center slices were larger than those for the isocenter slices, and dose differences in excess of 3\% were observed for the off-center slices of the rCBCT plan (see Figure 8(a) and Figure 8(c)). This shows that the variations in scatter contamination on each side of the cone-beam were large. The differences in excess of $3 \%$ between $\mathrm{RED}_{\text {гсвст }}$ and $\mathrm{RED}_{\text {simCT }}$ for the head-and-neck cases show that one electron density conversion table is not sufficient for sites with complex shapes. For the 10-cm off-center slice of the chest region, the $\mathrm{RED}_{\mathrm{rCBCT}}$ values for sites larger than the electron density phantoms were underestimated. Therefore, the calculated dose of the rCBCT plan for this site would be higher than that of the simCT plan (see Figure 8(f)). The passing rates of the gamma analysis $(2 \% / 2-\mathrm{mm}$ criterion) were less than $80 \%$

Table 3. Comparison of gamma passing rate with criterion of $2 \% \mathrm{DD}$ and 2-mm DTA for rCBCT plans and mCBCT plans.

\begin{tabular}{ccccc}
\hline & \multicolumn{2}{c}{ Prostate case } & \multicolumn{2}{c}{ Head-and-neck case } \\
\hline & rCBCT plan & mCBCT plan & rCBCT plan & mCBCT plan \\
\hline Axial_superior & $86.4 \% \pm 5.8 \%$ & $95.1 \% \pm 0.6 \%$ & $85.0 \% \pm 4.6 \%$ & $95.8 \% \pm 2.0 \%$ \\
Axial_center & $92.6 \% \pm 2.0 \%$ & $96.7 \% \pm 1.3 \%$ & $90.4 \% \pm 1.4 \%$ & $96.1 \% \pm 2.4 \%$ \\
Axial_inferior & $88.2 \% \pm 1.8 \%$ & $95.5 \% \pm 1.5 \%$ & $76.9 \% \pm 2.3 \%$ & $96.2 \% \pm 0.6 \%$ \\
Sagittal & $87.9 \% \pm 4.6 \%$ & $96.2 \% \pm 0.7 \%$ & $79.1 \% \pm 6.8 \%$ & $96.6 \% \pm 1.5 \%$ \\
\hline
\end{tabular}


(see Table 3). An electron density phantom of a chest should be used for this site. On the other hand, the $\mathrm{RED}_{\mathrm{mCBC}}$ values were in agreement with the REDsimct values, to within 3\%, for all of the slices (see Table 1 and Table 2), and the mCBCT plan produced doses that agree, to within $2 \%$, with those of the simCT plan (see Figure 8 and Table 3). Chu et al. [18] reported that a $20 \mathrm{HU}$ uncertainty in CT values for soft tissues and a $250 \mathrm{HU}$ uncertainty in CT values for bone result in no more than $2 \%$ of uncertainty in dose calculation. The results of dose calculation in this study are almost consistent with the results of their study.

In this study, we reduced the differences between the CBCT values and HUs using histograms of the CBCT values and HUs. Marchant et al. [17] have also described the histogram matching using the linear scaling. In their method, however, the differences in CBCT values for bone region were larger than soft tissue region because their method was global linear scaling of the CBCT values for the all regions. In this study, the linear scaling of CBCT values was performed for soft tissue and bone region because CBCT-ED conversion curve and HU-ED conversion curve have different gradients for the soft tissue and the bone region [10] [11]. In addition, this conversion method is independent of the differences in patient size, shapes, and inner tissue structures because this conversion method is performed for each slice.

Additional advantages of our method are that the patient's anatomical information for CBCT image acquisition is retained, in contrast to the pixel mapping method [9] and the bulk method [14] [15] [16]. The pixel mapping method changes the CBCT values to HUs after deformable image registration (DIR). However, this method depends on the accuracy of the DIR [4]. Therefore, inaccurate DIR may lead to changes in the anatomical information in CBCT images. The bulk method homogenizes CBCT values in regions of interest (ROIs). Therefore, the original heterogeneity of the ROIs may be lost. For the reasons stated above, our method can improve the accuracy of CBCT-ED conversion, which suggests improving the accuracy of dose calculations using CBCT images.

However, there are some limitations in our conversion method. As shown in Figure 7, it was difficult to discern adipose tissue and muscle in the histogram of the mCBCT image. This is due to the debasement of image contrast because of scatter contamination. In addition, the difference in the $\mathrm{RED}_{\mathrm{mCBCT}}$ of infraspinatus muscle for the $10-\mathrm{cm}$ off-center slice for the head-and-neck cases could have been caused by the effect of shading artifacts peculiar to the CBCT images. Further investigation is required to overcome this limitation because our method cannot eliminate this effect. Although our method can be applied to all treatment sites, there are some unresolved matters such as motion artifacts and differences in slice locations associated with breath. The mCBCT values and dose calculations for other locations will be studied in future research.

\section{Conclusions}

The method proposed herein can minimize the differences in the CBCT values 
in regions with different sizes, shapes, and inner tissue structures, as well as in off-center slices, because the СВCT values are converted using the histograms of each slice of simCT and CBCT images obtained for the same patient.

The proposed method yielded dose calculations from mCBCT images for prostate cancer and head-and-neck cancer cases that agreed very well, within $2.0 \%$, with those of simCT plans. Thus, the proposed method has considerable potential for clinical application in radiation treatment planning.

\section{Acknowledgements}

We wish to acknowledge valuable discussions with Professor Jun Takada, Assistant Professor Kenichi Tanaka, Associate Professor Kenichi Kamo, and Lecturer Toshihiro Kawaguchi of Sapporo Medical University. We would also like to thank the radiation oncologist and radiation technologists of KKR Sapporo Medical Center for their valuable comments on this paper.

\section{References}

[1] Oldham, M., Letourneau, D., Watt, L., Hugo, G., Yan, D., Lockman, D., Kim, L.H., Chen, P.Y., Martinez, A. and Wong, J.W. (2005) Cone-Beam-CT Guided Radiation Therapy: A Model for On-Line Application. Radiotherapy \& Oncology, 75, 271-278. https://doi.org/10.1016/j.radonc.2005.03.026

[2] Ingrosso, G., Miceli, R., Fedele, D., Ponti, E., Benassi, M., Barbarino, R., Murro, L.D., Giudice, E., Santarelli, F. and Santoni, R. (2012) Cone-Beam Computed Tomography in Hypofractionated Stereotactic Radiotherapy for Brain Metastases. Radiation Oncology, 7, 54.

http://ro-journal.biomedcentral.com/articles/10.1186/1748-717X-7-54 https://doi.org/10.1186/1748-717X-7-54

[3] Yoo, S. and Yin, F.F. (2006) Dosimetric Feasibility of Cone-Beam CT-Based Treatment Planning Compared to CT-Based Treatment Planning. International Journal of Radiation Oncology, Biology, Physics, 66, 1553-1561. https://doi.org/10.1016/j.ijrobp.2006.08.031

[4] Wu, Q.J., Li, T., Wu, Q. and Yin, F.F. (2011) Adaptive Radiation Therapy: Technical Components and Clinical Applications. Cancer Journal, 17, 182-189. https://doi.org/10.1097/PPO.0b013e31821da9d8

[5] Constantinou, C. and Harrington, J.C. (1992) An Electron-Density Calibration Phantom for CT-Based Treatment Planning Computers. Medical Physics, 19, 325-327. https://doi.org/10.1118/1.596862

[6] Thomas, S.J. (1999) Relative Electron Density Calibration of CT Scanners for Radiotherapy Treatment Planning. British Journal of Radiology, 72, 781-786. https://doi.org/10.1259/bjr.72.860.10624344

[7] Seco, J. and Evans, P.M. (2006) Assessing the Effect of Electron Density in Photon Dose Calculations. Medical Physics, 33, 540-552. https://doi.org/10.1118/1.2161407

[8] Hu, W., Ye, J., Wang, J., Ma, X. and Zhang, Z. (2010) Use of Kilovoltage X-Ray Volume Imaging in Patient Dose Calculation for Head-and-Neck and Partial Brain Radiation Therapy. Radiation Oncology, 5.

http://www.ro-journal.com/content/5/1/29 https://doi.org/10.1186/1748-717X-5-29

[9] Yang, Y., Schreibmann, E., Li, T., Wang, C. and Xing, L. (2007) Evaluation of On-Board 
$\mathrm{kV}$ Cone Beam CT (CBCT)-Based Dose Calculation. Physics in Medicine \& Biology, 52, 685-705. https://doi.org/10.1088/0031-9155/52/3/011

[10] Richter, A., Hu, Q., Steglich, D., Baier, K., Wilbert, J., Guckenberger, M. and Flentje, M. (2008) Investigation of the Usability of Conebeam CT Data Sets for Dose Calculation. Radiation Oncology, 3, 42.

http://ro-journal.biomedcentral.com/articles/10.1186/1748-717X-3-42 https://doi.org/10.1186/1748-717X-3-42

[11] Hatton, J., McCurdy, B. and Greer, P.B. (2009) Cone Beam Computerized Tomography: The Effect of Calibration of the Hounsfield Unit Number to Electron Density on Dose Calculation Accuracy for Adaptive Radiation Therapy. Physics in Medicine \& Biology, 54, N329-N346. https://doi.org/10.1088/0031-9155/54/15/N01

[12] Guan, H. and Dong, H. (2009) Dose Calculation Accuracy using Cone-Beam CT (CBCT) for Pelvic Adaptive Radiotherapy. Physics in Medicine \& Biology, 54, 6239-6250. https://doi.org/10.1088/0031-9155/54/20/013

[13] Rong, Y., Smilowitz, J., Tewatia, D., Tome, W.A. and Paliwal, B. (2010) Dose Calculation on $\mathrm{kV}$ Cone Beam CT Images: An Investigation of the HU-Density Conversion Stability and Dose Accuracy using the Site-Specipic Calibration. Medical Dosimetry, 35, 195-207.

[14] Van Zijtvetd, M., Dirkx, M. and Heijmen, B. (2007) Correction of Cone Beam CT Values Using a Planning CT for Derivation of the "Dose of the Day". Radiotherapy and Oncology, 85, 195-200.

[15] Boggula, R., Lorenz, F., Abo-Madyan, Y., Lohr, F., Wolff, D., Boda-Heggemann, J., Hesser, J., Wenz, F. and Wertz, H. (2009) A New Strategy for Online Adaptive Prostate Radiotherapy Based on Cone-Beam CT. Zeitschrift für Medizinische Physik, 19, 264-276.

[16] Fotina, I., Hopfgartner, J., Stock, M., Steininger, T., Lütgendorf-Caucig, C. and Georg, D. (2012) Feasibility of CBCT-Based Dose Calculation: Comparative Analysis of HU Adjustment Techniques. Radiotherapy and Oncology, 104, 249-256.

[17] Marchant, T.E., Moore, C.J., Rowbottom, C.G., Mackey, R.I. and Williams, P.C. (2008) Shading Correction Algorithm for Improvement of Cone Beam CT Images in Radiotherapy. Physics in Medicine \& Biology, 53, 5719-5733. https://doi.org/10.1088/0031-9155/53/20/010

[18] Chu, J.C.H., Ni, B., Kriz, R. and Saxena, V.A. (2000) Applications of Simulator Computed Tomography Number for Photon Dose Calculations during Radiotherapy Treatment Planning. Radiotherapy and Oncology, 55, 65-73. 